\title{
THE SOLAR SPECTRUM ON THE MARTIAN SURFACE AND ITS EFFECT ON PHOTOVOLTAIC PERFORMANCE
}

\author{
Geoffrey A. Landis ${ }^{1}$, Dan Hyatt ${ }^{2}$, and the MER Athena Science Team \\ ${ }^{1}$ NASA Glenn Research Center \\ 21000 Brookpark Road, M/S 302-1, Cleveland, OH 44135 \\ ${ }^{2}$ NASA SHARP Program \\ Elyria High School, Elyria, OH 44035
}

\begin{abstract}
Solar cells operating on the surface of Mars receive a spectrum of illumination different from the AMO spectrum, since the sunlight is filtered by dust suspended in the atmosphere. This spectrum changes with the amount of dust in the atmosphere, as well as with air mass change due to time of day and season. This spectral variation affects the performance of solar cells. We used data from Mars Exploration Rovers to measure this spectrum. By comparing the measured intensity with the known reflectance of the pancam calibration target on the rovers Spirit and Opportunity, we measure the solar spectrum reaching the surface. The effect of this spectrum on the performance of solar cells is then calculated based on the spectral response of several different solar cell types.
\end{abstract}

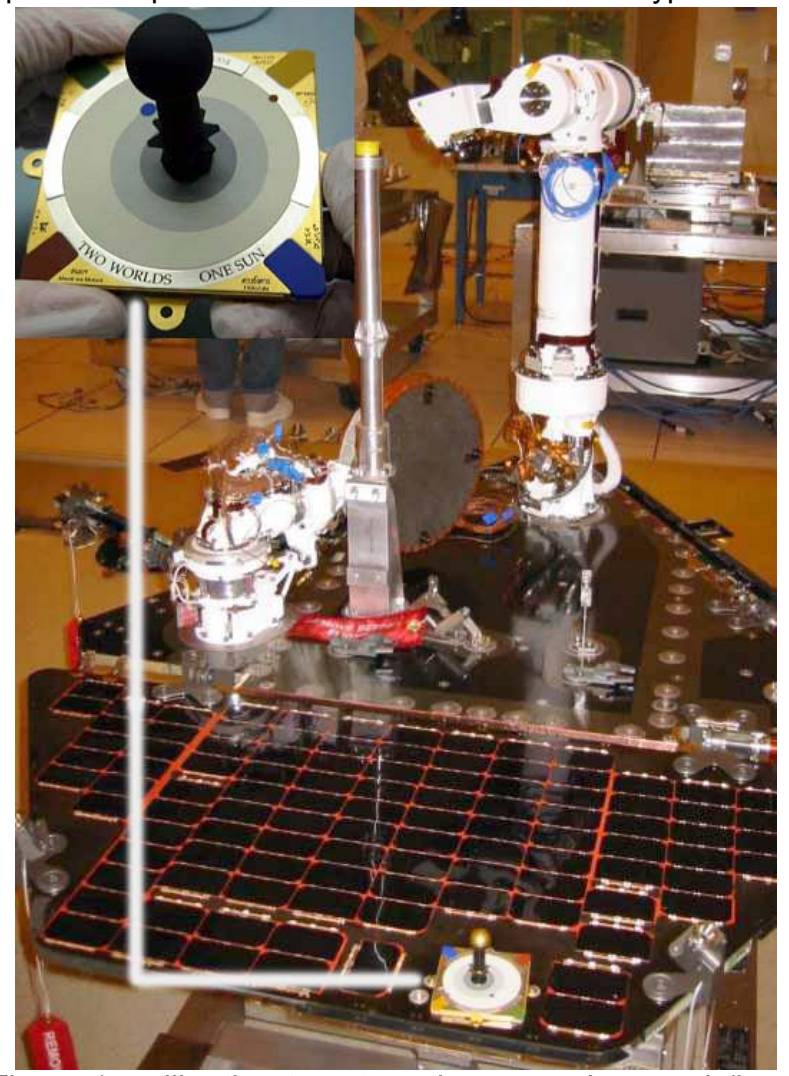

Figure 1: calibration target on the rear solar panel (inset shows target detail). The pancam is atop the white mast.

\section{INTRODUCTION}

Solar cells operating on the surface of Mars receive a different spectrum of illumination from the AMO spectrum, since the sunlight is filtered by dust suspended in the atmosphere. This spectrum changes with the amount of dust in the atmosphere, as well as with the change in air mass due to time of day and season [1,2]. In order to design solar arrays for the Martian surface [3], and to predict the performance during operation [4], it is desirable to measure the solar spectrum on the surface, and calculate how this affects solar cell performance

The twin rovers of the Mars Exploration Rovers mission, Spirit and Opportunity, landed on Mars in January 2003 [5]. One of the instruments of the MER mission is the MER Calibration Target. This calibration target consists of an $8 \mathrm{~cm} \times 8 \mathrm{~cm}$ target with a $6-\mathrm{cm}$ central shadow post, positioned on rear solar panel of both rovers (Figure 1). The target has three concentric rings, with nominal reflectivity of $60 \%, 40 \%$, and $20 \%$ respectively. The spectral characteristics of the calibration targets were measured before launch [6]. During the mission, these targets are repeatedly photographed by the Pancam instrument.

\section{METHOD}

The Pancam consists of two photometrically-calibrated cameras with silicon CCD-array focal planes, each with 8 narrow-band filters [6]. The radiatively calibrated ("RAD") dataset of the pancam allows each pixel to be converted into an absolute intensity to produce a value for the radiance in $\mathrm{W} / \mathrm{m}^{2}-\mathrm{nm}-\mathrm{sr}$. The absolute radiance calibration of the Pancam is within about $10 \%$, and the filter-to-filter precision of the reflectance measurements is about $3 \%$ or less [7]. Given the known reflectance of the calibration target [6] and the measured intensity of reflection, the intensity of sunlight on the calibration target can be used to calculate the actual incident intensity of the sunlight on Mars.

The calibration target includes a shadow pole used to produce a shadow on the calibration target. Figure 2 shows an example of the data set, showing the calibration target as viewed by the Pancam on Mars. Note that the shadow of the shadow post is not completely dark, indicating indirect (scattered) light. By comparing the incident intensity in the fully illuminated portion of the ring with the intensity of the same ring in a shadowed spot, the 
global and scattered intensity of sunlight can be retrieved; the direct component can then be calculated by subtraction. From this the transmission of the atmosphere is measured.

Since dust settles on the calibration target as it is exposed on the surface of Mars, the calibrated reflectance of the target drifts from the pre-flight value with time. The measurements of the calibration target used were from the first ten days after landing, before significant amounts of dust had settled.

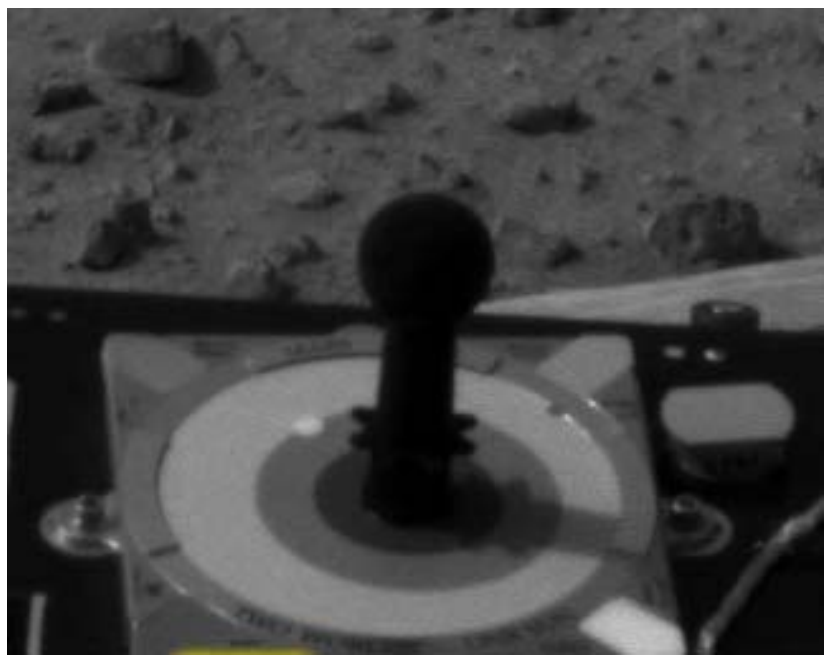

Figure 2: calibration target as viewed by the pancam on Mars (image courtesy of NASA/JPL and Cornell University).

\section{Solar intensity}

\section{RESULTS}

The amount of dust in the atmosphere is characterized by the optical depth ("tau"). The optical depth tau during Spirit's first week on Mars, when this data set used here was taken, was about 0.91 (measured in the blue, 440 $\mathrm{nm}$ ) to 0.95 (in the red, $980 \mathrm{~nm}$ ), while the optical depth during Opportunity's the first week on Mars was about 0.93 (at $440 \mathrm{~nm}$ ) to 0.95 (at $980 \mathrm{~nm}$ ) [10]. The optical depth decreased from this value during the primary mission, dropping to a value as low as 0.2 at the Spirit site in mid-winter.

The radiometrically-corrected data files from the MER Pancam investigation were retrieved from the NASA Planetary Data System [8]. The subset of calibration target images that contained the full filter set from the first ten sols after landing were selected from this data set. By comparing the measured intensity of the reflection from the calibration target with the pre-flight value, using standard radiometric calculations [9] the global, direct, and scattered intensity were found for various sun angles on both Spirit and Opportunity. Figures 3 shows the global solar intensity at the Spirit landing site, in Gusev Crater, as calculated from the intensity of the white ring of the cal target.

Likewise, figure 4 shows the global spectrum for the Opportunity landing site.

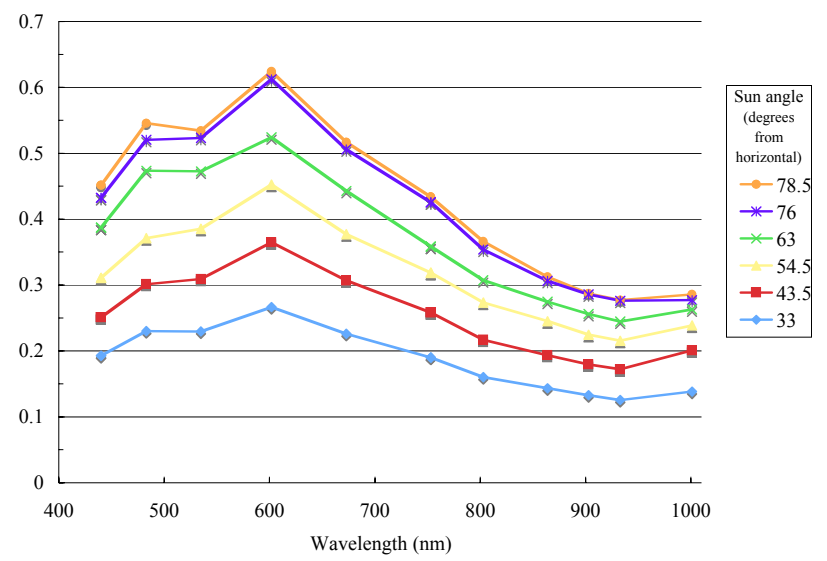

Figure 3: Global solar intensity at the Spirit landing site in Gusev Crater, plotted for wavelength 400 to $1000 \mathrm{~nm}$, for sun angles ranging from $33^{\circ}$ above the horizon (bottom curve) to $78.5^{\circ}$ above the horizon (top curve). Tau is approximately 0.93 for these curves.

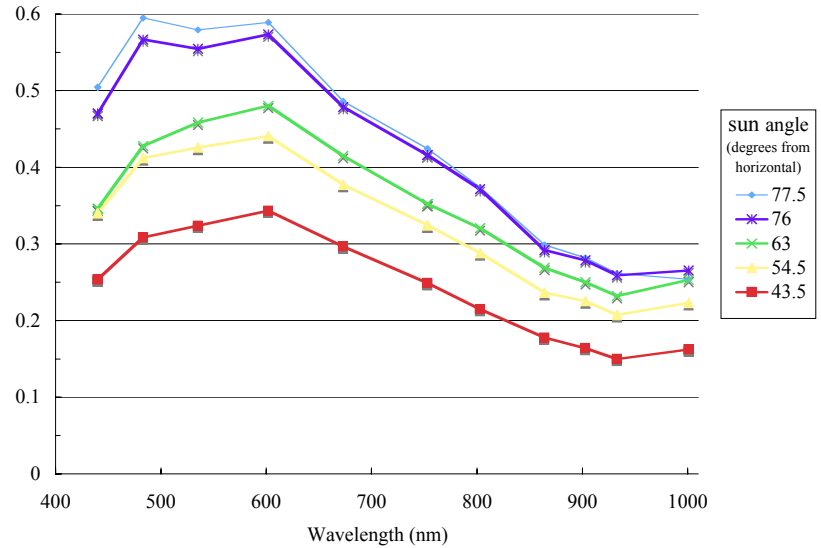

Figure 4: Global solar intensity at the Opportunity landing site in Meridiani Planum, plotted for wavelength 400 to $1000 \mathrm{~nm}$, for sun angles from $43^{\circ}$ (bottom curve) to $78^{\circ}$ (top curve). Tau is approximately 0.94 when these curves were measured.

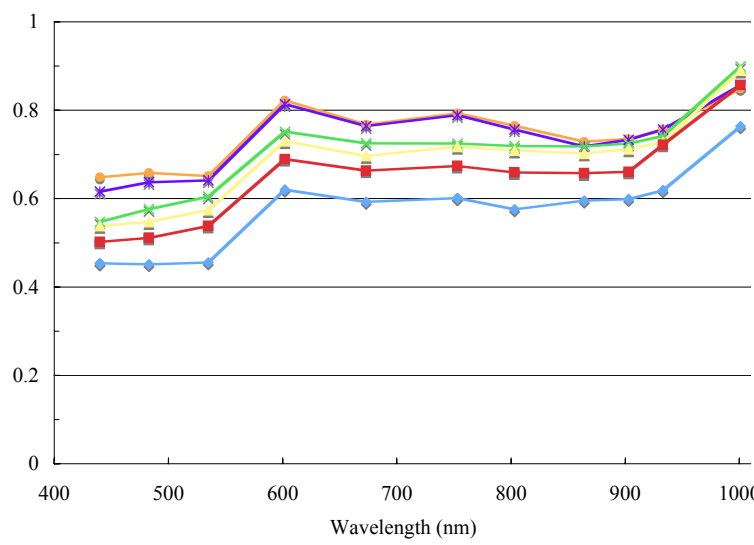

Figure 5: Atmospheric transmission for the global sunlight, $400 \mathrm{~nm}$ to $1000 \mathrm{~nm}$, for varied sun angles (averaged for Spirit and Opportunity data, tau approximately 0.94 ). 
By dividing this measured spectrum by the known spectrum outside the atmosphere, the fraction of light transmitted through the atmosphere can be calculated as a function of wavelength. This is shown in figure 5 . To smooth out the data, here the graph shown is the average of the Spirit and Opportunity transmission.

\section{Spectrum of Direct and Scattered Light}

The spectrum of the scattered ("indirect") component of the light can be measured using an identical technique, using the intensity of the shadowed area of the target. From this, the direct sunlight can be calculated by subtracting the scattered light from the global spectrum. The spectrum of the direct and scattered sunlight are compared in Figure 6. The scattered sunlight is very much enriched in long-wavelength ("red") light compared to the direct beam, as expected from the reddish color of the sky.

\section{EFFECT ON SOLAR CELL PERFORMANCE}

The effect of the atmospheric dust is separated into two overall effects: a decrease in overall intensity, and a spectrum shift. The intensity as a function of dust optical depth has been covered elsewhere [3].

The spectral effect is calculated as a spectral factor equal to the Isc of the cell (or subcell) under Martian spectrum, divided by the Isc under AM0 illumination of the same intensity. Factors greater than one indicate improved performance under Martian spectrum.

The short circuit current of the cell under Martian and AMO illumination is calculated by integration of the spectrum times the cell spectral response, using spectral response of test cells measured at NASA Glenn.

Spectral factor was calculated for a number of cell types. Of significant interest is the performance of a GalnP solar cell, since this is the top subcell of the threejunction cell [11], and is the cell which limits the shortcircuit current. The measured spectral response of this cell is shown in figure 7. For comparison, we also measured a GaAs cell, both as a single-junction GaAs solar cell, and also as a GaAs cell as the middle element of a tandem with a GalnP top cell. [labeled GaAs(mid)] . The GalnP filter serves to remove the blue part of the spectrum, wavelengths less than about $700 \mathrm{~nm}$, from the light reaching the GaAs cell.

The $\mathrm{Ge}$ subcell of the multijunction cell was not measured, since the wavelength of peak response of $\mathrm{Ge}$ is beyond the range of spectral measurements made by the MER Pancam. Since the Ge subcell is never the current-limiting junction, knowledge of the Ge performance under Mars illumination is not required.

These were compared with a silicon cell, which is responsive to light of wavelength as long as $1000 \mathrm{~nm}$ table 1.

The calculated spectral shift factors are tabulated in

As expected, the cells with the highest response in the blue degraded in performance under the red-rich Martian spectrum, and the amount of degradation increased as the sun angle approached the horizontal. The cells with the highest response to red and infrared improved in performance, on the other hand, had improved performance under Mars illumination. The change is slightly greater for sun angles near the horizon, where the incident light travels through a greater airmass and hence is filtered more by the reddish dust. The cell with the greatest improvement in performance was the filtered GaAs cell, since the GalnP filter removed the blue portion of the spectrum.

Hence, under Martian illumination, the top (GalnP) subcell of the multi-cell stack is the cell which limits the short-circuit current of the three-cell tandem stack. Since the GalnP short-circuit current decreases by as much as $13.8 \%$ (at the lowest sun angle, 33 degrees), while the middle cell increases by $2.8 \%$, if the subcells were matched in current at AM0, the mismatch under highangle Mars sunlight is as much as $16.6 \%$.

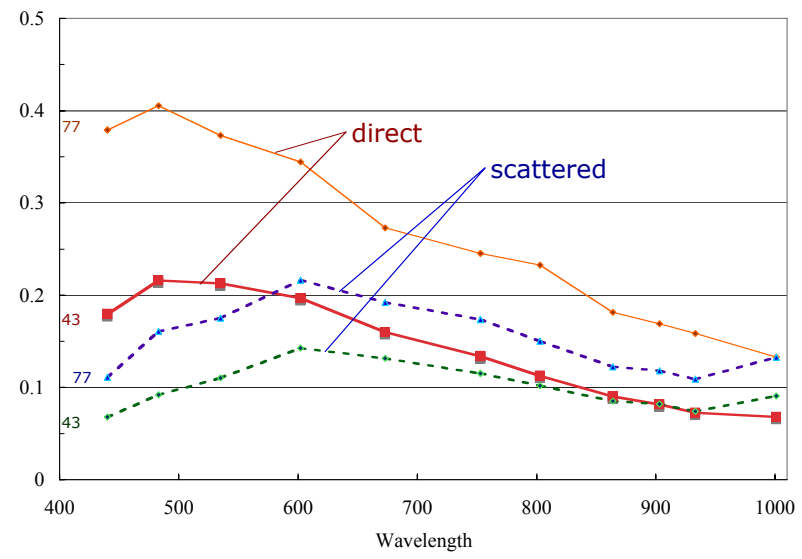

Figure 6: Comparison of the spectrum of direct and scattered sunlight on the Spirit calibration target at wavelength from 400 to $1000 \mathrm{~nm}$, for sun angles of 43 and 78 degrees above the horizontal (tau approximately 0.93 ).

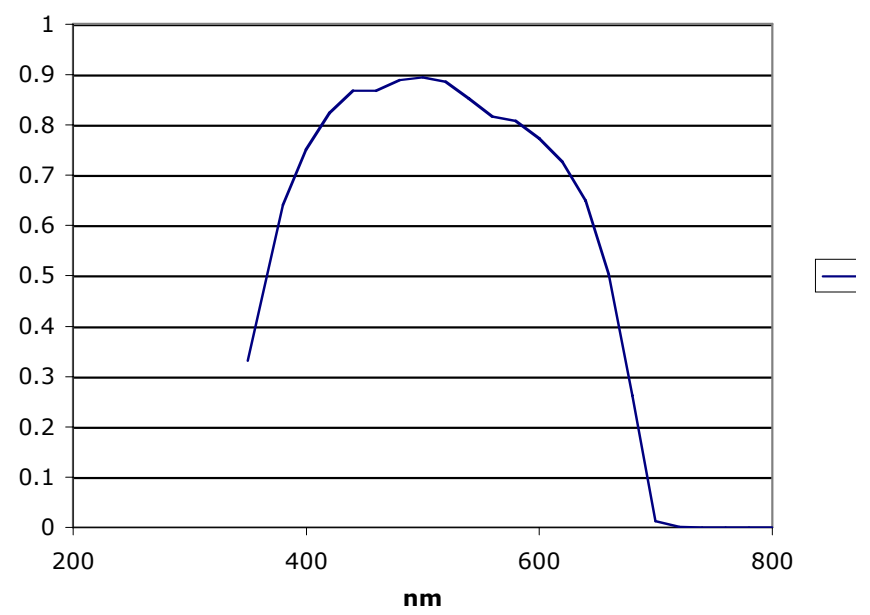

Figure 7: Spectral response (expressed as external quantum yield, QY) of the GalnP top cell of the threejunction cell type used on the MER rover. 


\begin{tabular}{|l|c|c|c|r|r|r|}
\hline & \multicolumn{6}{|c|}{ Solar Elevation (degrees above horizon) } \\
Cell Type & $\underline{33}$ & $\underline{44}$ & $\underline{55}$ & $\underline{63}$ & $\underline{76}$ & $\underline{78.5}$ \\
\hline InGaP(top) & 86.2 & 84.9 & 85.3 & 81.4 & 90.7 & 90.9 \\
\hline GaAs & 94.1 & 93.2 & 93.8 & 93.1 & 97.2 & 97.2 \\
\hline GaAs(mid) & 102.8 & 102.2 & 103.1 & 101.6 & 103.8 & 103.7 \\
\hline Si & 100.4 & 100.5 & 100.4 & 100.5 & 100.4 & 100.5 \\
\hline
\end{tabular}

Table 1: Solar cell spectral factors (in \%) at the Spirit site (tau approximately 0.93 ) for Mars sunlight, for various sun angles.

\section{ACKNOWLEDGEMENTS}

We would like to acknowledge the MER Athena Science team, and in particular the Pancam team for the radiometrically calibrated data, and Dave Scheiman for the spectral response data. Subsequent to this analysis of the initial few sols of the mission, a much more detailed model of spectrum for the extended MER mission was put together by Bell, Savransky, and Wolff of the MER Athena Pancam team [12], examining the color of the sky as a function of the amount of the dust in the atmosphere.

\section{REFERENCES}

[1] R. Haberle, et al., "Atmospheric Effects on the Utility of Solar Power on Mars," Resources of Near Earth Space, University of Arizona Press, 1993, pp. 799-818.

[2] G. Landis, "Solar Cell Selection for Mars," IEEE Aerospace and Electronic Systems Magazine, Vol. 15, No. 1, Jan. 2000, pp. 17-21. Presented at the 2nd World Conference on Photovoltaic Energy Conversion, Vol. III, Vienna, Austria, July 1998, pp. 3695-3698.

[3] G. Landis and J. Appelbaum, "Design Considerations for Mars PV Power Systems," Proceedings of the 21st
IEEE Photovoltaic Specialists Conference, Vol. 2, May 1990, pp. 1263-1270.

[4] R. Ewell and D.R. Burger, "Solar Array Model Corrections from Mars Pathfinder Lander Data," Proceedings of the 26th IEEE Photovoltaic Specialists Conference, Sept.-Oct. 1997, pp. 1019-1022.

[5] G. Landis, "Exploring Mars with Solar-Powered Rovers," Proc. 31st. IEEE Photovoltaic Specialist's Conference, Orlando FL, Jan 3-7 2005, pp. 858-861.

[6] J. F. Bell, III, et al., "The Mars Exploration Rover Athena Panoramic Camera (Pancam) Investigation," Journal of Geophysical Research, 108 (E12), doi:10.1029/2003JE002070, 2003, p. 8063.

[7] J. F. Bell, III, J. Joseph, J. N. Sohl-Dickstein, H. M. Arneson, M.J. Johnson, M. T. Lemmon, and D. Savransky "In-flight Calibration and Performance of the Mars Exploration Rover Panoramic Camera (Pancam) Instruments," Journal of Geophysical Research, 111, E02S03, doi:10.1029/2005JE002444 (2006).

[8] NASA, Planetary Data System (PDS): MER data files are found at the MER Analyst's Notebook, http://anserver1.eprsl.wustl.edu/

[9] W. L. Wolfe, Introduction to Radiometry, International Society for Optical Engineering., Bellingham, WA, 1998.

[10] M. Lemmon, et al., "Atmospheric Imaging Results from the Mars Exploration Rovers," Science, Vol. 360, No. 5702, Dec. 3 2004, pp. 1753-1756.

[11] P. Stella, R. Ewell1 and J. Hoskin, "Design and Performance of the MER (Mars Exploration Rovers) Solar Arrays," Proc. 31st. IEEE Photovoltaic Specialist's Conference, Orlando FL, Jan 3-7 2005, pp. 626-630.

[12] J. F. Bell III, D. Savransky, and M. J. Wolff, "Chromaticity of the Martian Sky as Observed by the Mars Exploration Rover Pancam Instruments," Journal of Geophysical Research (in press). 